\title{
Regioselective one-pot, three component synthesis of ethyl 6-aryl-3- propylpyridazine-4-carboxylates in water
}

\author{
Jabbar Khalafy $^{a^{*}}$, Mehdi Rimaz ${ }^{\mathrm{b}}$ and Mahnaz Ezzati ${ }^{\mathrm{a}}$
}

${ }^{a}$ Chemistry Department, Urmia University, P.O. Box 57154, Urmia, Iran ${ }^{b}$ Department of Chemistry, Payame Noor University, PO Box 19395-3697, Tehran, Iran

ART I C LE I N F O

Article history:

Received March 30, 2012

Received in Revised form

May 21, 2012

Accepted 14 June 2012

Available online

14 June 2012

Keywords:

Pyridazine

Arylglyoxal

Hydrazine

Regioselective

\author{
A B S T R A C T \\ A regioselective synthesis of ethyl 6-aryl-3-propylpyridazine-4-carboxylates by one-pot three- \\ component reaction of ethyl butyrylacetate with various arylglyoxals in the presence of \\ hydrazine hydrate at room temperature in water was described.
}

\section{Introduction}

Chemical processes that allow assembly of several building blocks in a single operation are gaining increasing attention in the search for new efficient, diversity-oriented synthetic methodologies, and particularly those directed toward heterocyclic compounds. Such processes are highly desirable for the rapid generation of libraries of small drug-like molecules for high-throughput screaning. ${ }^{1-6}$ The design of multicomponent syntheses often relies on the integration of multiple individual reactions to give a one-pot synthetic operation, a new concept with important economical and environmental issues. ${ }^{7-10}$

The chemistry and pharmacology of pyridazine derivatives have recently received considerable interest. This can be realized from the vast number of articles and patents published in the synthesis, 
chemistry, and biological activities of pyridazines. ${ }^{11-14}$ The pyridazines and their derivatives exhibit a broad range of biological activity, such as analgesic, ${ }^{15}$ antibacterial, ${ }^{16}$ anti-inflammatory, ${ }^{11}$ antihypertensive $^{17}$ or antihistaminic ${ }^{18}$ properties. The derivatives of pyridazines could also find application as ligands in metallic complexes which possess catalytic properties. ${ }^{19,20}$ These compounds could also be used as semi-conductor materials and as materials with non-linear optical properties. ${ }^{21}$ These pharmacological and technological properties of pyridazines encourage the development of methods for their synthesis and functionalization. In continuation of our interest in the synthesis of various pyridazine derivatives, ${ }^{22-26}$ we decided to investigate the three-component reaction of arylglyoxals, ethyl butyrylacetate and hydrazine hydrate which is extended to the regioselective synthesis of ethyl 6-aryl-3-propylpyridazine-4-carboxylates as a new series of three substituted pyridazines.

\section{Results and Discussion}

Similar to our latest researches describing the synthesis of pyridazine derivatives, ${ }^{22-26}$ firstly the arylglyoxals (1-6) were produced from the corresponding acetophenones via oxidation with $\mathrm{SeO}_{2}$ in dioxane at reflux conditions. ${ }^{27}$ Then the reaction of arylglyoxals (1-6) with ethyl butyrylacetate (7) in the presence of hydrazine hydrate at room temperature in water leds to form the ethyl 6-aryl-3propylpyridazine-4-carboxylates (8-14) in good yields as shown in the Scheme 1.

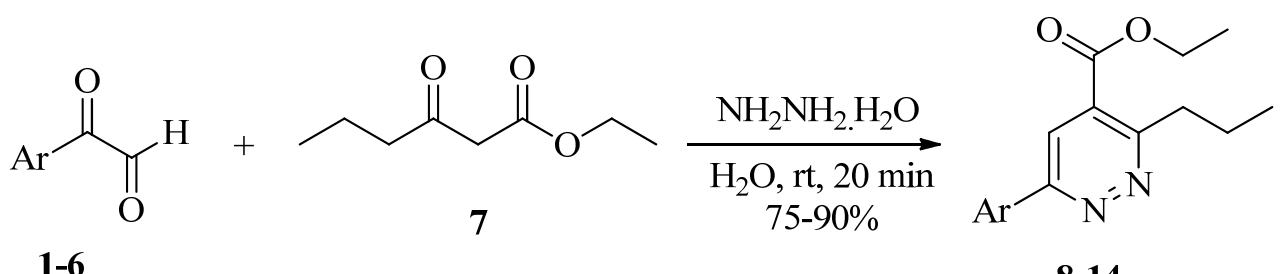

1-6<smiles>COc1ccc(C)cc1OC</smiles>

Scheme 1: Synthesis of ethyl 6-aryl-3-propylpyridazine-4-carboxylates

All of the obtained products were listed at the Table 1. Formation of the 6-aryl substituted pyridazines as sole products proves this fact that the outlined strategy acts regioselectively and there is no evidence for production of 5-aryl substituted isomers. The mechanism of the regioselective synthesis of the products was shown in Scheme 2.

Owing to the high reactivity of the external carbonyl in comparison with internal carbonyl in an arylglyoxal system, the reaction was run through the nucleophilic attack of enol (15) to the aldehyde carbonyl of arylglyoxal (6) leading to form the intermediate (16) as a sole product. The subsequent condensation of hydrazine with the carbonyl groups of the intermediate (16) gives the final product (13). 
Table 1. List of pyridazine products.

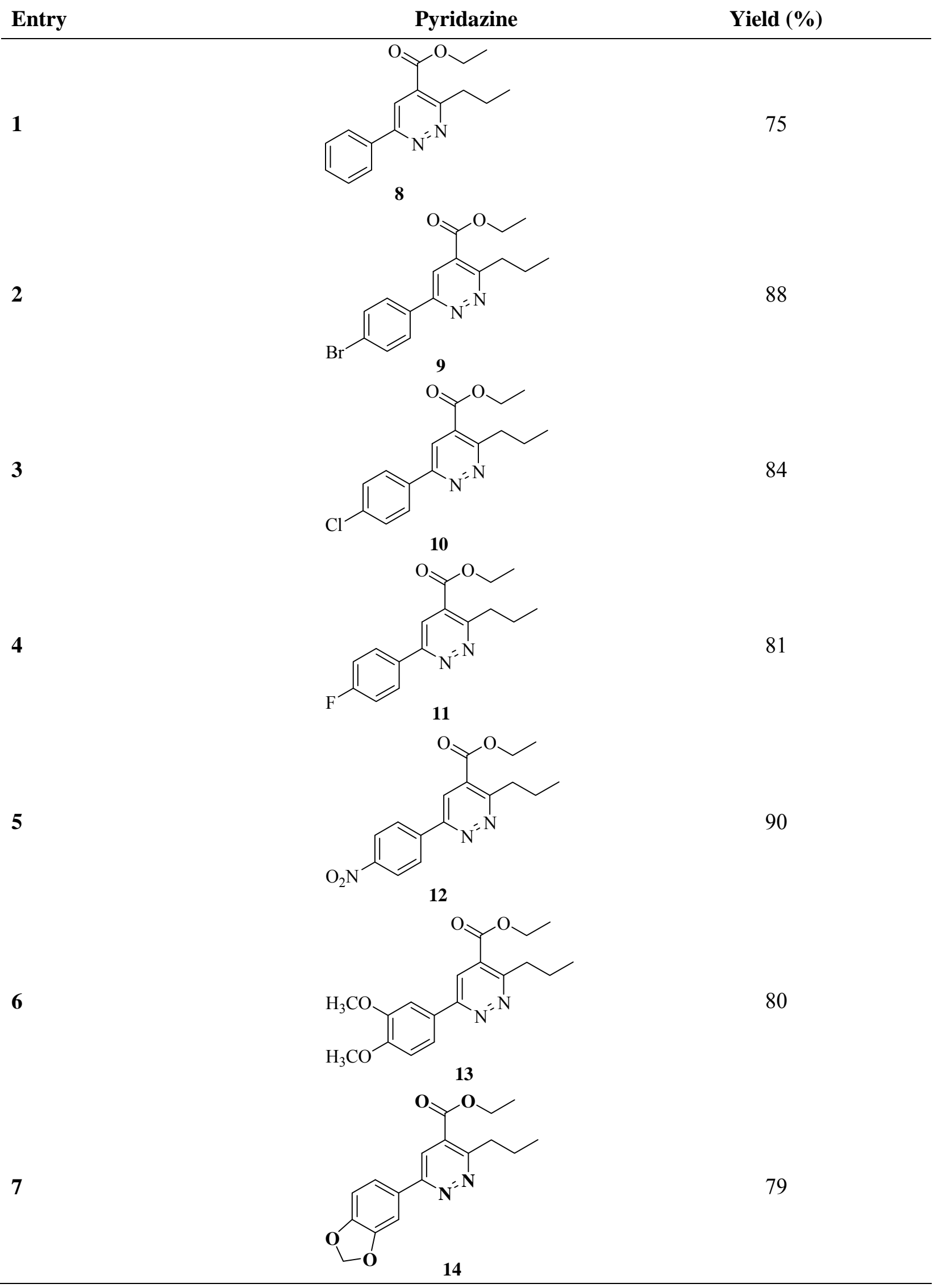



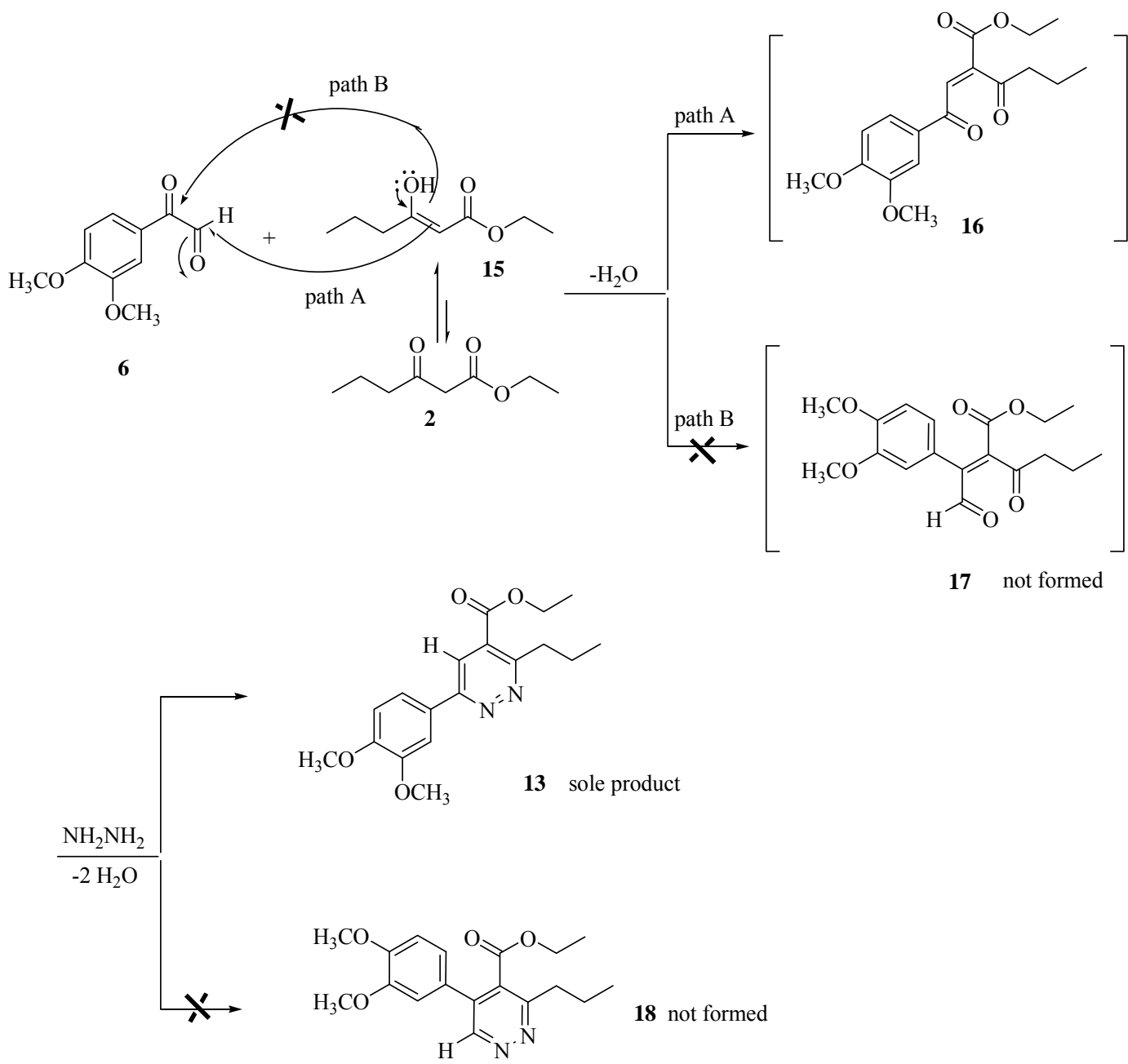

Scheme 3: Suggested mechanism for regioselective synthesis of ethyl 6-(3,4-dimethoxyphenyl)-3propylpyridazine-4-carboxylate

\section{Conclusions}

We have synthesized some new three substituted pyridazines via one-pot three component reaction of arylglyoxals, ethyl butyrylacetate and hydrazine hydrate in water at room temperature. The simple operation, the high regioselectivity and the convenient reaction condition of this procedure makes it a valuable route to prepare new substituted pyridazines.

\section{Acknowledgements}

The authors express their thanks to the research council of Urmia University for financial support.

\section{Experimental}

\section{General Procedures}

Elemental analyses for $\mathrm{C}, \mathrm{H}$ and $\mathrm{N}$ were performed using Heraeus Leco analyzer 932. NMR spectra were recorded on a Bruker Avance spectrometer operating at $300 \mathrm{MHz}$ for ${ }^{1} \mathrm{H}$ nuclei and $75.5 \mathrm{MHz}$ for ${ }^{13} \mathrm{C}$ nuclei with tetramethylsilane as internal standard. Mass spectra were recorded using a Varian 
Matt 311 spectrometer operating at nominal accelerating voltage of $70 \mathrm{eV}$. Thin layer chromatography (TLC) was run on silica percolated aluminium plates (Merck Kieselgel F254). Melting points were determined on a Kofler hot-stage apparatus.

Sample procedure for pyridazine synthesis.

To a mixture of ethyl butyrylacetate $(1 \mathrm{mmol})$ and $\operatorname{arylglyoxal}^{27}(1 \mathrm{mmol})$ in water $(5 \mathrm{~mL})$, was successively added hydrazine hydrate $(5 \mathrm{mmol})$ at room temperature; the resultant mixture was stirred for 20-35 min during which time a precipitate was formed. The precipitate was then filtered and washed with excess water. The crude product was purified by recrystallization from ethanol.

Ethyl 6-phenyl-3-propylpyridazine-4-carboxylate (8): yellow crystals, $75 \%$, mp $50-51^{\circ} \mathrm{C}$.

FT-IR (KBr) $v_{\max }: 3064,2958,2868,1725,1648,1582,1451,1394,1240,1093,755,690 \mathrm{~cm}^{-1} .{ }^{1} \mathrm{H}-$ NMR (300 MHz, $\left.\mathrm{CDCl}_{3}\right) \delta(\mathrm{ppm}): 8.27(\mathrm{~s}, 1 \mathrm{H}, \mathrm{Ar}), 8.10-8.16(\mathrm{~m}, 2 \mathrm{H}, \mathrm{Ar}), 7.50-7.58(\mathrm{~m}, 3 \mathrm{H}, \mathrm{Ar})$, 4.49 (q, J $7.2 \mathrm{~Hz}, 2 \mathrm{H}, \mathrm{CH}_{2}$ ), 3.41 (t, J $7.2 \mathrm{~Hz}, 2 \mathrm{H}, \mathrm{CH}_{2}$ ), 1.87 (sex, J 7.2 Hz, 2H, CH ), 1.47 (t, J 7.2 $\left.\mathrm{Hz}, 3 \mathrm{H}, \mathrm{CH}_{3}\right), 1.08\left(\mathrm{t}, \mathrm{J} 7.2 \mathrm{~Hz}, 3 \mathrm{H}, \mathrm{CH}_{3}\right) .{ }^{13} \mathrm{C}-\mathrm{NMR}\left(75 \mathrm{MHz}, \mathrm{CDCl}_{3}\right) \delta(\mathrm{ppm}): 164.07,160.38$, 158.79, 134.02, 132.67, 131.18, 129.32, 127.19, 126.73, 62.96, 34.82, 23.48, 14.13, 14.05. Anal. Calcd for $\mathrm{C}_{16} \mathrm{H}_{18} \mathrm{~N}_{2} \mathrm{O}_{2}, \mathrm{C} 71.09, \mathrm{H}$ 6.71, N 10.36; Found: C, 71.13; H, 6.66; N, 10.50. Mass spectrum m/z (\%): 270 ([M $\left.\left.\mathrm{M}^{+}\right], 18\right), 242$ (100), 241 (82), 213 (36), 197 (37), 170 (88).

Ethyl 6-(4-bromophenyl)-3-propylpyridazine-4-carboxylate (9): yellow crystals, 88\%, mp 66-68 ${ }^{\circ} \mathrm{C}$. FT-IR (KBr) $v_{\max }: 3070,2959,2935,2872,1706,1597,1505,1404,1388,1223,1156,851,574 \mathrm{~cm}^{-1}$. ${ }^{1} \mathrm{H}-\mathrm{NMR}\left(300 \mathrm{MHz}, \mathrm{CDCl}_{3}\right) \delta(\mathrm{ppm}): 8.25$ (s, 1H, Ar), $8.02(\mathrm{~d}, \mathrm{~J} 8.1 \mathrm{~Hz}, 2 \mathrm{H}, \mathrm{Ar}), 7.68$ (d, J 8.1 Hz, 2H, Ar), 4.49 (q, J 7.2 Hz, 2H, $\mathrm{CH}_{2}$ ), 3.41 (t, J 7.2 Hz, 2H, $\mathrm{CH}_{2}$ ), 1.86 (sex, J 7.2 Hz, 2H, $\mathrm{CH}_{2}$ ), 1.46 (t, J 7.2 Hz, 3H, $\left.\mathrm{CH}_{3}\right), 1.07\left(\mathrm{t}, \mathrm{J} 7.2 \mathrm{~Hz}, 3 \mathrm{H}, \mathrm{CH}_{3}\right) .{ }^{13} \mathrm{C}-\mathrm{NMR}\left(75 \mathrm{MHz}, \mathrm{CDCl}_{3}\right) \delta(\mathrm{ppm}): 164.71$, $160.63,157.41,133.78,132.43,130.01,128.49,125.46,124.84,62.74,35.76,23.30,14.17,14.09$. Anal. Calcd for $\mathrm{C}_{16} \mathrm{H}_{17} \mathrm{BrN}_{2} \mathrm{O}_{2}$, C 55.03, H 4.91, N 8.02; Found: C, 55.05; H, 4.99; N, 8.11.

Ethyl 6-(4-chlorophenyl)-3-propylpyridazine-4-carboxylate (10): yellow crystals, 84\%, mp 69-70 ${ }^{\circ} \mathrm{C}$. FT-IR (KBr) $v_{\max }: 3068,2963,2931,2871,1734,1594,1414,1385,1238,1095,1010,850,756 \mathrm{~cm}^{-1}$. ${ }^{1} \mathrm{H}$ NMR $\left(300 \mathrm{MHz}, \mathrm{CDCl}_{3}\right) \delta(\mathrm{ppm}): 8.16(\mathrm{~s}, 1 \mathrm{H}, \mathrm{Ar}), 8.07$ (d, J $\left.8.4 \mathrm{~Hz}, 2 \mathrm{H}, \mathrm{Ar}\right), 7.51$ (d, J $8.4 \mathrm{~Hz}$, 2H, Ar), 4.47 (q, J 7.2 Hz, 2H, CH 2$), 3.35$ (t, J 7.2, 2H, $\mathrm{CH}_{2}$ ), 1.85 (sex, J 7.2 Hz, 2H, $\mathrm{CH}_{2}$ ), 1.45, (t, J $\left.7.2 \mathrm{~Hz}, 3 \mathrm{H}, \mathrm{CH}_{3}\right), 1.05\left(\mathrm{t}, \mathrm{J} 7.2 \mathrm{~Hz}, 3 \mathrm{H}, \mathrm{CH}_{3}\right) .{ }^{13} \mathrm{C}-\mathrm{NMR}\left(75 \mathrm{MHz}, \mathrm{CDCl}_{3}\right) \delta(\mathrm{ppm}): 165.24,160.50$, 157.06, 136.63, 133.90, 129.34, 129.15, 128.15, 123.72, 62.48, 36.25, 23.15, 14.16, 14.08. Anal. Calcd for $\mathrm{C}_{16} \mathrm{H}_{17} \mathrm{ClN}_{2} \mathrm{O}_{2}$, C 63.05, H 5.62, N 9.19; Found: C, 63.00; H, 5.56; N, 9.20. Mass spectrum m/z (\%): 306 ([M $\left.\left.{ }^{+}+2\right], 3\right), 304$ ([ $\left.\left.\mathrm{M}^{+}\right], 10\right), 276$ (100), 247 (42), 231 (40), 206 (32), 204 (89), 152 (19), 139 (46), $75(20)$.

Ethyl 6-(4-fluorophenyl)-3-propylpyridazine-4-carboxylate (11): pale yellow crystals, 81\%, mp $42-43^{\circ} \mathrm{C}$.

FT-IR (KBr) v $v_{\max }: 3055,2963,2873,1716,1602,1509,1389,1254,1163,1094,845,757,552 \mathrm{~cm}^{-1}$. ${ }^{1} \mathrm{H}-\mathrm{NMR}\left(300 \mathrm{MHz}, \mathrm{CDCl}_{3}\right) \delta(\mathrm{ppm}): 8.16(\mathrm{~s}, 1 \mathrm{H}, \mathrm{Ar}), 8.08-8.15(\mathrm{~m}, 2 \mathrm{H}, \mathrm{Ar}), 7.18-7.27$ (m, $\left.2 \mathrm{H}, \mathrm{Ar}\right)$, 4.48 (q, J $7.2 \mathrm{~Hz}, 2 \mathrm{H}, \mathrm{CH}_{2}$ ), 3.36 (t, J 7.2, 2H, CH 2 ), 1.86 (sex, J 7.2 Hz, 2H, CH $\left.3 \mathrm{H}, \mathrm{CH}_{3}\right), 1.08\left(\mathrm{t}, \mathrm{J} 7.2 \mathrm{~Hz}, 3 \mathrm{H}, \mathrm{CH}_{3}\right) .{ }^{13} \mathrm{C}-\mathrm{NMR}\left(75 \mathrm{MHz}, \mathrm{CDCl}_{3}\right) \delta(\mathrm{ppm}): 165.92,165.30,162.60$, $160.21,157.15,131.60,128.92$, 128.81, 123.68, 116.30, 116.01, 62.44, 36.21, 23.14, 14.14, 14.07. Anal. Calcd for $\mathrm{C}_{16} \mathrm{H}_{17} \mathrm{FN}_{2} \mathrm{O}_{2}, \mathrm{C}, 66.65$, H 5.94, N 9.72; Found: C, 65.72; H, 5.90; N, 9.85. Mass spectrum m/z (\%): 288 ([M $], 38), 260$ (100), 231 (37), 215 (45), $188(90), 157$ (13), 120 (29).

Ethyl 6-(4-nitrophenyl)-3-propylpyridazine-4-carboxylate (12): orange crystals, $90 \%, \mathrm{mp} 118^{\circ} \mathrm{C}$. FT-IR (KBr) $v_{\max }: 3078,2965,2931,2872,1733,1601,1514,1394,1346,1305,1242,1093,1019$, 857, 753, $694 \mathrm{~cm}^{-1} .{ }^{1} \mathrm{H}-\mathrm{NMR}\left(300 \mathrm{MHz}, \mathrm{CDCl}_{3}\right) \delta(\mathrm{ppm}): 8.41(\mathrm{~d}, \mathrm{~J} 8.7 \mathrm{~Hz}, 2 \mathrm{H}, \mathrm{Ar}), 8.33$ (d, J 8.7 Hz, 2H, Ar), 8.27 (s, 1H, Ar), 4.50 (q, J 7.2 Hz, 2H, $\mathrm{CH}_{2}$ ), 3.40 (t, J 7.2 Hz, 2H, CH 2 ), 1.87 (sex, J 7.2 
$\left.\mathrm{Hz}, 2 \mathrm{H}, \mathrm{CH}_{2}\right), 1.48\left(\mathrm{t}, \mathrm{J} 7.2 \mathrm{~Hz}, 3 \mathrm{H}, \mathrm{CH}_{3}\right), 1.08\left(\mathrm{t}, \mathrm{J} 7.2,3 \mathrm{H}, \mathrm{CH}_{3}\right) .{ }^{13} \mathrm{C}-\mathrm{NMR}\left(75 \mathrm{MHz}, \mathrm{CDCl}_{3}\right) \delta$ (ppm): 164.83, 161.56, 156.14, 149.06, 141.26, 129.49, 127.84, 124.54, 124.30, 62.74, 36.21, 23.19, 14.15, 14.05. Anal. Calcd for $\mathrm{C}_{16} \mathrm{H}_{17} \mathrm{~N}_{3} \mathrm{O}_{4}, \mathrm{C}$ 60.94, H 5.43, N 13.33; Found: $\mathrm{C}, 60.92 ; \mathrm{H}, 5.39$; N, 13.43. Mass spectrum m/z (\%): 315 ([ $\left.\left.\mathrm{M}^{+}\right], 7\right), 287$ (93), 258 (59), 242 (44), 215 (100), 152 (27), 139 (28), 115 (19), 75 (18).

Ethyl 6-(3,4-dimethoxyphenyl)-3-propylpyridazine-4-carboxylate (13): yellow crystals, 80\%, mp 64-65 ${ }^{\circ} \mathrm{C}$.

FT-IR (KBr) $v_{\max }: 3087,2958,2935,2873,2835,1716,1590,1516,1465,1399,1261,1222,1149$, 1095, 1020, 872, $762 \mathrm{~cm}^{-1} .{ }^{1} \mathrm{H}-\mathrm{NMR}\left(300 \mathrm{MHz} \mathrm{CDCl}_{3}\right) \delta(\mathrm{ppm}): 8.26(\mathrm{~s}, 1 \mathrm{H}, \mathrm{Ar}), 7.93(\mathrm{~s}, 1 \mathrm{H}, \mathrm{Ar})$, 7.57 (d, J $8.1 \mathrm{~Hz}, 1 \mathrm{H}, \mathrm{Ar}), 7.01$ (d, J $8.1 \mathrm{~Hz}, 1 \mathrm{H}, \mathrm{Ar}), 4.50$ (q, J $\left.7.2 \mathrm{~Hz}, 2 \mathrm{H}, \mathrm{CH}_{2}\right), 4.01(\mathrm{~s}, 3 \mathrm{H}$, $\left.\mathrm{OCH}_{3}\right), 3.97$ (s, 3H, $\mathrm{OCH}_{3}$ ), 3.39 (t, J 7.2, 2H, CH 2 ), 1.87 (sex, J 7.2 Hz, 2H, CH $\left.3 \mathrm{H}, \mathrm{CH}_{3}\right), 1.07$ (t, J 7.2, 3H, CH3). ${ }^{13} \mathrm{C}-\mathrm{NMR}\left(75 \mathrm{MHz}, \mathrm{CDCl}_{3}\right) \delta(\mathrm{ppm}): 163.57,159.80,158.27$, 152.40, 150.01, 132.21, 127.72, 125.56, 120.55, 111.22, 109.88, 63.24, 56.37, 56.11, 34.22, 23.49, 14.14, 14.02. Anal. Calcd for $\mathrm{C}_{18} \mathrm{H}_{22} \mathrm{~N}_{2} \mathrm{O}_{4}, \mathrm{C}$ 65.44, H 6.71, N 8.48; Found: C, 65.37; H, 6.68; N, 8.57. Mass spectrum m/z (\%): 330 ([M $\left.\left.\mathrm{M}^{+}\right], 26\right), 302$ (100), 301 (55), 273 (24), 257 (25), 230 (82), 204 (24), 165 (32).

Ethyl 6-(benzo[d][1,3]dioxol-5-yl)-3-propylpyridazine-4-carboxylate (14): yellow crystals, 79\%, mp 89-90 ${ }^{\circ} \mathrm{C}$.

FT-IR (KBr) $v_{\max }: 3083,2961,2928,1725,1499,1446,1402,1254,1036,875 \mathrm{~cm}^{-1} .{ }^{1} \mathrm{H}-\mathrm{NMR}(300$ $\left.\mathrm{MHz}, \mathrm{CDCl}_{3}\right) \delta(\mathrm{ppm}): 8.20(\mathrm{~s}, 1 \mathrm{H}, \mathrm{Ar}), 7.71(\mathrm{~s}, 1 \mathrm{H}, \mathrm{Ar}), 7.62(\mathrm{~d}, \mathrm{~J} 7.8 \mathrm{~Hz}, 1 \mathrm{H}, \mathrm{Ar}), 6.96$ (d, J 7.8 Hz, $1 \mathrm{H}, \mathrm{Ar}), 6.08$ (s, 2H, $\left.\mathrm{CH}_{2}\right), 4.49$ (q, J $\left.7.2 \mathrm{~Hz}, 2 \mathrm{H}, \mathrm{CH}_{2}\right), 3.40$ (t, J 7.2, 2H, $\left.\mathrm{CH}_{2}\right), 1.84$ (sex, J 7.2 Hz, $\left.2 \mathrm{H}, \mathrm{CH}_{2}\right), 1.46$ (t, J 7.2 Hz, 3H, $\left.\mathrm{CH}_{3}\right), 1.07$ (t, J 7.2, 3H, $\left.\mathrm{CH}_{3}\right) .{ }^{13} \mathrm{C}-\mathrm{NMR}\left(75 \mathrm{MHz}, \mathrm{CDCl}_{3}\right) \delta(\mathrm{ppm})$ : $164.86,159.84,158.17,151.85,149.02,133.07,131.53,127.21,122.14,108.95,107.28,101.93$, 63.11, 34.43, 23.48, 14.15, 14.06. Anal. Calcd for $\mathrm{C}_{17} \mathrm{H}_{18} \mathrm{~N}_{2} \mathrm{O}_{4}, \mathrm{C}$ 64.96, H 5.77, N 8.91; Found: C, $64.90 ; \mathrm{H}, 5.75 ; \mathrm{N}, 8.86$.

\section{References}

1. Zhu J. (2003) Recent developments in the isonitrile-based multicomponent synthesis of heterocycles. Eur. J. Org. Chem., 68, 1133-1144.

2. Bienayme H., Hulme C., Oddon G., and Schmitt P. (2000) Maximizing synthetic efficiency: Multi-component transformations lead the way. Chem. Eur. J., 6, 3321-3329.

3. Domling A., and Ugi I. (2000) Multicomponent reactions with isocyanides. Angew. Chem., Int. Ed., 39, 3168-3210.

4. Weber L., Illgen K., and Almstetter M. (1999) Discovery of new multi component reactions with combinatorial methods. Synlett, 366- 374.

5. Dax S. L., McNally J. J., and Youngman M. A. (1999) Multi-component methodologies in solidphase organic synthesis, Curr. Med. Chem, 6, 255-270.

6. Armstrong R. W., Combs A. P., Tempest P. A., Brown S. D., and Keating T. A. (1996) Multiplecomponent condensation strategies for combinatorial library synthesis, Acc. Chem. Res., 29, 123131.

7. Orita A., Yoshioka N., Struwe P., Braiser A., Beckmann A., and Otera J. (1999) Integrated chemical process: one-pot double elimination method for acetylenes. Chem. Eur. J., 5, 13551363.

8. Grigg R., Liu A., Shaw D., Sunganthan S., Washington M.,Woodall D., and Yoganathan G. (2000) Palladium-catalysed cascade molecular queuing-cycloaddition, cyclocondensation and Diels-Alder reactions. Tetrahedron Lett., 41, 7129-7133.

9. Braun R., Zeitter K., and Müller T. (2001) A novel one-pot pyrrole synthesis via a couplingisomerization-stetter-paal-knorr sequence, Org. Lett., 3, 3297-3300. 
10. Clique B., Vassiliou S., Monteiro N., and Balme G. (2002) Integrated transition metal catalyzed reactions: synthesis of polysubstituted 4-(phenoxymethyl)-3-pyrrolines and their isomers by onepot coupling of propargylamines, vinyl sulfones (or nitroalkenes) and phenols, Eur. J. Org. Chem., 1493-1499.

11. Tamayo N., Liao L., Goldberg M., Powers D., Tudor Y. Y., Yu V., Wong L. M., Henkle B., Middleton S., Syed R., Harvey T., Jang G., Hungate R., and Dominguez, C. (2005) Design and synthesis of potent pyridazine inhibitors of p38 MAP kinase. Bioorg. Med. Chem. Lett., 15, 2409-2413.

12. Nagawade R. R., Khanna V. V., Bhagwat S. S., and Shinde D. B. (2005) Synthesis of new series of 1-Aryl-1,4-dihydro-4-oxo-6-methyl pyridazine-3-carboxylic acid as potential antibacterial agents. Eur. J. Med. Chem., 40, 1325-1330.

13. Sotelo E., and Ravina E. (2002) Pyridazine derivatives. XXIV. [1] efficient n-methylation of diversely substituted 3(2H)-pyridazinones using N,N-dimethylformamide dimethylacetal, Synth. Commun, 32, 1675-1680.

14. Coelho A. Sotelo E. Novoa H. Peeters O. M. Blaton N. and Ravina E. (2004) Pyridazine derivatives. Part 38: Efficient Heck alkenylation at position 5 of the 6-phenyl-3(2H)pyridazinone system, Tetrahedron, 45, 3459-3463.

15. Rohet F. Rubat C. Coudert P., and Couquelet J. (1997) Synthesis and analgesic effects of 3substituted 4,6-diarylpyridazine derivatives of the arylpiperazine class. Bioorg. Med. Chem., 5, 655-659.

16. Tucker J. A. Allwine D. A. Grega K. C. Barbachyn M. R. Klock J. L. Adamski J. L. Brickner S. J. Hutchinson D. K. Ford C. W. Zurenko G. E. Conradi R. A. Burton P. S. and Jensen R. M. (1998) Piperazinyl oxazolidinone antibacterial agents containing a pyridine, diazene, or triazene heteroaromatic ring, J. Med. Chem, 41, 3727-3735.

17. Benson, S. C., Palabrica C. A., and Snyder J. K. (1987) Indole as a dienophile in inverse electron demand Diels-Alder reactions. 5H-Pyridazino[4,5-b]indoles as cycloadducts with 3,6dicarbomethoxy-1,2,4,5-tetrazine, J. Org. Chem., 52, 4610-4614.

18. Gyoten M., Nagaya H., Fukuda S., Ashida Y., and Kawano Y. (2003) Synthesis of eosinophil infiltration inhibitors with antihistaminic activity, Chem. Pharm. Bull., 51, 122-133.

19. Sung N. D., Yung K. S., Kim T. Y., Choi K. Y., Such M., Kim J. G., Suh I. H., and Chin, J. (2001) Synthesis and characterization of a novel tetranuclear nickel(II) complex: $\left[\mathrm{Ni}_{4}(\mu-\mathrm{OH})_{2}(\mu-\right.$ dppn $\left.)_{4}\left(\mu-\mathrm{H}_{2} \mathrm{O}\right)_{2}\right](\mathrm{Cl})\left(\mathrm{ClO}_{4}\right)_{5} 9 \mathrm{H}_{2} \mathrm{O}$ (dppn=3,6-bis(2'-pyridyl)pyridazine). Inorg. Chem. Commun., 4, 377-380.

20. Brooker S., Davidson T. C., Hay S. J., Kelly R. J., Kennepohl D. K., Plieger P. G., Moubaraki B., Murray K. S., Bill E., and Bothe E. (2001) Doubly pyridazine-bridged macrocyclic complexes of copper in $+1,+2$ and mixed valent oxidation states, Coord. Chem. Rev., 216-217, 3-30.

21. Cheng Y., Ma B. and Wudl F. (1999) Synthesis and optical properties of a series of pyrrolopyridazine derivatives: deep blue organic luminophors for electroluminescent devices, $J$. Mater. Chem., 9, 2183-2188.

22. Rimaz M., and Khalafy J. (2010) Novel one-pot, three component synthesis of alkyl 6-aryl-3methylpyridazine-4-carboxylates in water. Arkivoc, ii, 110-117.

23. Rimaz M., Khalafy J., and Najafi Moghadam, P. (2010) A regioselective one-pot, three component synthesis of 6-aryl-4-cyano-3(2H)-pyridazinones in water. Aust. J. Chem, 63, 13961401.

24. Rimaz M., Khalafy J., Noroozi Pesyan N., and Prager R. H. (2010) A simple one-pot, three component synthesis of 3-arylpyrimido[4,5-c]pyridazine-5,7(6H,8H)-diones and their sulfur analogues as potential monoamine oxidase inhibitors. Aust. J. Chem., 63, 507-510.

25. Rimaz M., Noroozi Pesyan N., and Khalafy J. (2010) Tautomerism and isotopic multiplets in the ${ }^{13} \mathrm{C}$ NMR spectra of partially deuterated 3-arylpyrimido[4,5-c]pyridazine-5,7 $(6 \mathrm{H}, 8 \mathrm{H})$-diones and 
their sulfur analogs-evidence for elucidation of the structure backbone and tautomeric forms, Magn. Reson. Chem. 48, 276-285.

26. Khalafy J., Rimaz M., Panahi L., and Rabiei H. (2011). A regiospecific one-pot, three component synthesis of 4-aryl-6,8-dimethylpyrimido[4,5-c]pyridazine-5,7 $(6 \mathrm{H}, 8 \mathrm{H})$-diones as new potential monoamine oxidase inhibitors, Bull. Korean Chem. Soc., 32, 2428-2432.

27. Riley H. A., and Gray A. R. (1943) Phenylglyoxal. Organic Syntheses, Wiley \& Sons: New York, NY, Collect. Vol. II, p 509. 